\title{
REGISTROS NOTABLES DE MURCIÉLAGOS EN EL ESTADO DE OAXACA, MEXICO
}

\author{
JOSÉ L. GARCÍA-GARCÍA, ANA M. ALFARO E. Y ANTONIO SANTOS-MORENO \\ Laboratorio de Ecología Animal. Centro Interdisciplinario de Investigación para \\ el Desarrollo Integral Regional, Unidad Oaxaca, Instituto Politécnico Nacional, Hornos \\ 1003, Santa Cruz Xoxocotlán, Oaxaca, Oaxaca, C. P. 71230. \\ asantosm90@hotmail.com
}

\begin{abstract}
The range of five species of bats (Enchisthenes hartii, Anoura geoffroyi, Hylonycteris underwoodi, Rhogeessa tumida and Myotis californica) is extended from new records for localities in the districts of Choapam and Juchitán, Oaxaca, Mexico, of specimens collected in 2004 and 2005.
\end{abstract}

Keywords: Chiroptera, bat record, Oaxaca, range extension.

Palabras clave: Chiroptera, registros de murciélagos, Oaxaca, distribución.

El estado de Oaxaca, en el sureste de México, es una entidad sobresaliente por su riqueza de especies en varios grupos animales, incluidos los mamíferos, de los que hasta la fecha se ha documentado la presencia de 192 especies (Alfaro et al., 2005), distribuidas en siete familias (Briones-Salas y Sánchez-Cordero, 2004). En este número destacan los murciélagos que representan el $60.87 \%$ de las 138 especies registradas en el país (Ceballos et al., 2002). A pesar de esta notable riqueza, aún existen zonas muy amplias por inventariar en el Estado, por lo que la distribución de las especies dentro de la entidad aún es incompleta. El objetivo de este trabajo es presentar información adicional sobre la distribución de algunas especies de murciélagos en los distritos de Choapam y Juchitán. Se consideró como registro notable cuando la nueva localidad se localiza en un distrito en el que previamente no se tenía conocimiento de la presencia de la especie. En todos los casos los ejemplares se capturaron con redes de niebla colocadas cerca de fuentes de agua, senderos hechos por humanos o animales dentro de la vegetación. La determinación de la especie se realizó con claves de Álvarez et al. (1994) y Medellín et al. (1997). De cada organismo capturado se muestran las medidas corporales convencionales: longitud total, de la cola vertebral, de la pata derecha y de la oreja (mm) y el peso (grs). Asimismo se muestran también algunas medidas craneales, también en mm: longitud mayor del cráneo (LOMC), longitud condilobasal (LOCB), anchura de la caja craneal (ANCC), anchura interorbital posterior (ANINPO), longitud de la hilera maxilar de dientes (LOHMD) y longitud palatal (LOPA) (De Blase y Martín, 1979). Los números de catalogo que se mencionan son los del preparador (AS-M). 
Enchisthenes hartii (Thomas, 1892)

El 8 de mayo de 2005 se colectó una hembra (número de catálogo 444). Medidas somáticas: 64, 0, 11, 15 y 12 g de peso. Medidas craneales LOMC 10.10, LOCB 10.56, ANCC 9.21, ANINPO 4.42, LOHMD 6.32 y LOPA 8.67. El sitio de colecta fue $4.6 \mathrm{~km} \mathrm{~S}$

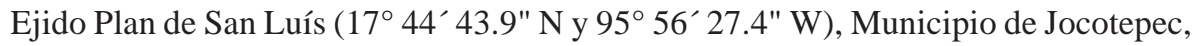
Distrito de Choapam, a una altitud de $106 \mathrm{~m}$, en la zona conocida como la Chinantla baja. La vegetación es selva mediana. La localidad conocida más cercana se ubica en el Distrito de Juchitan, Sierra Madre, al norte de Zanatepec (Goodwin, 1969). No se pudo georreferenciar el registro histórico debido a su ambigüedad. Se estima que el aumento en el área de distribución en el estado es poco más de 200 km. Esta especie se encuentra incluida en la Norma Oficial Mexicana en la categoría de Protección especial. Otras especies registradas en la localidad fueron Balantiopteryx plicata, Glossophaga soricina, Artibeus jamaicensis, Centurio senex y Sturnira lilium.

\section{Anoura geoffroyi Gray, 1838}

El 18 de julio de 2004 se colectó un macho con testículos escrotados (número de catálogo 232). Sus medidas somáticas: 62, 0, 9, 12 y 16 g de peso. Medidas craneales LOMC 24.5, LOCB 10.16, ANCC 9.50, ANINPO 4.89, LOHMD 9.33 y LOPA 13.45. La localidad de colecta es $0.8 \mathrm{~km}$ E Benito Juárez (16 42' 45.4'” $\mathrm{N}$ y 94ㅇ 8' 23.1'” W), Municipio de San Miguel Chimalapa, Distrito de Juchitán, a 930 m de altitud, en la zona de los Chimalapas. En el sitio la vegetación predominante es bosque de pino-encino. La localidad más cercana de ocurrencia de la especie es San Gabriel Mixtepec, Sola de Vega (Goodwin, 1969). La nueva localidad representa una extensión de $315 \mathrm{~km}$ en el área de distribución. Las otras especies registradas fueron Sturnira lilium, S. ludovici, Artibeus jamaicensis, A. intermedius y Carollia perspicillata.

\section{Hylonycteris underwoodi Thomas, 1903}

El 25 de enero 2005 se colectó un macho (número de catálogo 327). Sus medidas somáticas son 62, 7, 9, 8 y 10 g de peso. Medidas craneales LOMC 21.42, LOCB 8.60, ANCC 8.34, ANINPO 3.76, LOHMD 7.81 y LOPA 13.33. La localidad de colecta es 6 km NW Benito Juárez (16²4' 20"N y 94 11’ 45"W), Municipio de San Miguel Chimalapa, Distrito de Juchitán, $1700 \mathrm{~m}$ de altitud. El tipo de vegetación es un ecotono de bosque mesófilo de montaña y selva alta. La localidad más cercana previamente conocida es San José Chacalapa en el distrito de Yautepec (Goodwin, 1969). La nueva localidad representa una ampliación de $208 \mathrm{~km}$ para el área de distribución en el Estado. Otras especies registradas fueron Glossophaga soricina, Dermanura tolteca, Uroderma bilobatum, Artibeus jamaicensis, Sturnira lilium, Carollia sowelli y Eptesicus brasiliensis. 
Este último ejemplar representa también el primer registro de la especie para el estado de Oaxaca (García-García et al., enviado).

\section{Rhogeessa tumida H. Allen,1866}

El 28 de octubre de 2004 se colectó una hembra (numero de catalogo 274), con medidas somáticas: 76, 30, 5, 11 y 7 g de peso. Medidas craneales: LOMC 13.47, LOCB 6.90, ANCC 6.03, ANINPO 3.79, LOHMD 5.00 y LOPA 6.58. La localidad de colecta es $0.8 \mathrm{~km}$ E Benito Juárez (16² 42’ 45.4"N y 94 8’ 23.1"W), Municipio de San Miguel Chimalapa, Distrito de Juchitán, a 930 m de altitud. La vegetación predominante es bosque de pino-encino. La localidad más cercana previamente conocida es el municipio de Tehuantepec, en el distrito de Tehuantepec (Goodwin, 1969). La nueva localidad representa una ampliación de $124 \mathrm{~km}$. Otras especies registradas fueron Carollia sowelli, Glossophaga soricina y Dermanura tolteca.

\section{Myotis californica (Audubon \& Bachean, 1842)}

El 21 de enero de 2005 se colectó una hembra (número de catalogo 321). Medidas somáticas: 41, 39, 5, 10 y 5 g de peso. Medidas craneales: LOMC 13.07, LOCB 6.77, ANCC 6.28, ANINPO 3.62, LOHMD 5.19 y LOPA 6.70. La localidad de colecta es $0.8 \mathrm{~km}$ E Benito Juárez (16² 42’ 45.4"N y 94 8’ 23.1"W), Municipio de San Miguel Chimalapa, Distrito de Juchitán, a 930 m de altitud. La vegetación predominante es bosque de pino-encino. La localidad más cercana previamente conocida es Santo Domingo Chontecomatlán, en el municipio de Santa Maria Ecatepec en el distrito de Yautepec (Goodwin, 1969). La nueva localidad representa una ampliación de $207 \mathrm{~km}$ del área de distribución. Otras especies registradas fueron Glossophaga soricina, Tadarida brasiliensis y Uroderma bilobatum.

\section{AGRADECIMIENTOS}

A la asociación Ecoprodes S. C. por invitar a los autores a participar en el proyecto de biodiversidad desarrollado en el distrito de Tuxtepec y a las autoridades del municipio de San Miguel Chimalapa. A la Coordinación General de Estudios de Posgrados e Investigación (CGPI) del Instituto Politécnico Nacional por el apoyo económico.

\section{LITERATURACITADA}

Alfaro, A.M., J.L. García-García y A. Santos-Moreno. 2005. The false vampire bat Vampyrum spectrum in Oaxaca, México. Bat Research News, 46:145-146. 
Álvarez, T., S.T. Álvarez-Castañeda y J.C. López Vidal. 1994. Claves para murciélagos mexicanos. Instituto Politécnico Nacional. México.

Briones-Salas, M. y V. Sánchez-Cordero. 2004. Mamíferos. Pp. 423-447, en: Biodiversidad de Oaxaca (A. J. García Mendoza, M. J. Ordóñez y M. Briones-Salas eds. y coords.), Instituto de Biología, UNAM-Fondo Oaxaqueño para la Conservación de la NaturalezaWold Wildlife Found. México.

Ceballos, G., J. Arroyo-Cabrales y R.A. Medellín. 2002. The mammals of México: composition, distribution, and conservation. Occasional Papers The Museum Texas Tech University, 218:1-27.

De Blase, A.F. y R.E. Martín. 1979. Manual of Mammalogy, Anatomy and Natural History. W. M. C. Browm Co. Publishers.

García-García, J.L., A. Santos-Moreno y A. Ma. Alfaro . en prensa. First record of Eptesicus brasiliensis in Oaxaca, México. Bat Research News.

Goodwin, G. 1969. Mammals from the State of Oaxaca, Mexico in the American Museum of Natural History. Bulletin of the American Museum of Natural History, 141:1-269.

Medellín, R.A., H.T. Arita y O. Sánchez. 1997. Guía de identificación de los murciélagos de México. Comisión Nacional para el Conocimiento de la Biodiversidad. México. 